\title{
Does emotional memory enhancement assist the memory-impaired?
}

\author{
Lucas S. Broster ${ }^{1}$, Lee X. Blonder ${ }^{1,2,3}$ and Yang Jiang ${ }^{1,2,4 *}$ \\ ' Department of Behavioral Science, University of Kentucky College of Medicine, Lexington, KY, USA \\ ${ }^{2}$ Sanders-Brown Center on Aging, University of Kentucky College of Medicine, Lexington, KY, USA \\ ${ }^{3}$ Department of Neurology, University of Kentucky College of Medicine, Lexington, KY, USA \\ ${ }^{4}$ Magnetic Resonance Imaging and Spectroscopy Center, Lexington, KY, USA
}

\author{
Edited by: \\ P. Hemachandra Reddy, Oregen \\ Health and Science University, USA \\ Reviewed by: \\ P. Hemachandra Reddy, Oregen \\ Health and Science University, USA \\ Qitao Ran, University of Texas \\ Health Science Center at San \\ Antonio, USA \\ *Correspondence: \\ Yang Jiang, Department of \\ Behavioral Science, Aging, Brain, \\ and Cognition Laboratory, University \\ of Kentucky College of Medicine, \\ 113 Medical Behavioral Science \\ Building, Lexington, KY 40536-0086, \\ USA. \\ e-mail: yjiang@uky.edu
}

We review recent work on emotional memory enhancement in older adults and patients with mild cognitive impairment (MCl) or Alzheimer dementia (AD) and evaluate the viability of incorporating emotional components into cognitive rehabilitation for these groups. First, we identify converging evidence regarding the effects of emotional valence on working memory in healthy aging. Second, we introduce work that suggests a more complex role for emotional memory enhancement in aging and identify a model capable of unifying disparate research findings. Third, we survey the neuroimaging literature for evidence of a special role for the amygdala in $\mathrm{MCl}$ and early $\mathrm{AD}$ in emotional memory enhancement. Finally, we assess the theoretical feasibility of incorporating emotional content into cognitive rehabilitation given all available evidence.

Keywords: aging, Alzheimer disease, mild cognitive impairment, cognitive rehabilitation, amygdala, emotional memory enhancement, executive function, working memory

\section{INTRODUCTION}

Contemporary cognitive rehabilitation, especially when delivered alongside pharmacotherapy, has been shown to improve memory outcomes and delay dementia progression in patients with mild cognitive impairment (MCI) and early Alzheimer dementia (AD) (Mimura and Komatsu, 2007). Historically, however, clinical results of cognitive dementia interventions have been somewhat mixed. In fact, the findings of trials investigating the efficacy of such practices in the 1980s led clinicians to question the validity of the entire behavioral intervention paradigm for the treatment of AD (Hopper, 2003; Mowszowski et al., 2010).

These early cognitive rehabilitation interventions focused on improving explicit memory as a means of treating the explicit memory loss typical of AD. Other forms of memory, including implicit memory, are relatively spared by AD. Implicit memory includes those aspects of memory of which the individual lacks conscious awareness. For example, procedural memory, the memory of how to perform physical tasks, is one aspect of implicit memory. Repetition learning, the passive, automatic learning of associations upon repeated exposure, is another (Kessels et al., 2011).

The advent of implicit memory-based methods represented a major theoretical shift in the development of more efficacious cognitive rehabilitation protocols. Rather than focusing only on improving explicit memory directly through rehearsal, these protocols also utilize intact implicit memory to compensate for eroding explicit capacity (Mimura and Komatsu, 2007). Pilot studies have suggested improved efficacy for implicit memorybased intervention methods (Zanetti et al., 1997; Kessels and de
Haan, 2003; van Halteren-van Tilborg et al., 2007; Jean et al., 2010a,b).

The success of this approach leads us to question whether other neurocognitive systems broadly implicated in memory are relatively intact in patients with progressive dementia such that those systems may be analogously harnessed to help compensate for eroded explicit capacity. In other words, much as implicit memory treatment appears promising for ameliorating early explicit memory decline, perhaps recruiting other relatively intact memory processes in individuals with early impairment can additively help delay significant functional impairment. One candidate system that involves structures relatively spared in $\mathrm{AD}$ is the emotional processing system, and its integration with cognitive memory systems has garnered significant attention over the past decades (for reviews, refer to Pessoa, 2008, 2010). To evaluate whether this system might show promise in further enhancing cognitive rehabilitation protocols, we first describe how emotional memory enhancement, the tendency of emotional content to be better-remembered than non-emotional content, might change with aging and dementia.

\section{EMOTIONAL MEMORY ENHANCEMENT IS INTACT IN OLDER ADULTS}

A major behavioral basis for the belief that emotional content might improve memory outcomes is the persistent finding that, even in older adults, emotional content is remembered more accurately and/or more quickly than non-emotional content for both short and longer-term recall of visual images (Moayeri et al., 2000; Boller et al., 2002; Hiroaki, 2002; Mikels et al., 2005; 
Redondo and Fernández-Rey, 2010; Borg et al., 2011; EvansRoberts and Turnbull, 2011; Nashiro et al., 2011). This is independently demonstrated for emotional stimuli of both positive valence and negative valence relative to non-emotional control, and it is demonstrated in research participants with early $\mathrm{AD}$ (Boller et al., 2002; Borg et al., 2011).

In tests simultaneously evaluating positively and negatively valenced stimuli relative to non-emotional control, both types consistently increase memory performance better than nonemotional stimuli, and, for older adults, positively valenced stimuli tend to produce better results than negatively valenced stimuli (Nashiro et al., 2011; for a meta-analysis of positivity effects in younger and older adults, see Murphy and Isaacowitz, 2008). The tendency for older adults to perform better on positively valenced stimuli with regard to measures of attention, recognition, and emotional memory enhancement has been termed the age-related positivity effect (Charles et al., 2003; Mather and Knight, 2006; Löckenhoff and Carstensen, 2007; Grühn et al., 2007; Isaacowitz et al., 2009). The positivity effect phenomenon itself is sufficiently well established that separate attempts to model the phenomenon compete for legitimacy in the emotional aging literature (for separate accounts refer to Carstensen et al., 1999; Rozin and Royzman, 2001; Labouvie-Vief, 2003; Mather and Knight, 2005; Knight et al., 2007; or to Labouvie-Vief et al., 2007; Scheibe and Carstensen, 2010).

One account of the positivity effect holds that changes in valence biases reflect top-down changes in emotional regulation with aging (Carstensen et al., 1999). In other words, it appears that older adults may have unique mental processes that enable them to encode positively valenced stimuli in a manner categorically different from younger adults (Charles and Carstensen, 2007). This perspective suggests that older adults may have special valence-specific cognitive biases that may be used in the context of emotional memory enhancement to improve memory outcomes.

This positivity effect may not be observed in some subpopulations of older adults. For example, individuals with major depressive disorder or who have recovered from major depressive disorder show preferential attention to negatively valence stimuli, which may predispose them to encode valence differently from individuals without a history of depression (Kerestes et al., 2012). Whether these effects persist in the face of the positivity effect is unknown, but, given the association between depression and cognitive impairment, the competing trends should be noted in the short-term and formally evaluated in future work (for a meta-analysis, see Burt et al., 1995; for a full review of the interaction between these conditions, see Korczyn and Halperin, 2009).

For the purposes of this review, the observations that older adults show emotional memory enhancement, particularly with positively valenced stimuli, and that research participants with $\mathrm{AD}$ may also show a degree of emotional memory enhancement are sufficient for motivating further inquiry. We next look at how emotional memory enhancement differs in old age and dementia.

\section{DEMENTIA CHANGES THE EMOTIONAL REALM}

While emotional memory enhancement, as prior discussed, is intact to a certain extent in older adults, individuals with MCI, and individuals with $\mathrm{AD}$, all of these groups do significantly differ from younger adults on certain attention and memory measures (reviewed by Kensinger, 2009). The prior-discussed positivity effect between younger and older adults is just one example of such a difference, and the themes implicit in that effect are broadly consistent even for non-visual modalities, attention paradigms, and neurofunctional work (Leclerc and Kensinger, 2011; Orgeta, 2011; Yang and Hasher, 2011). However, differences in emotional memory enhancement between non-demented older adults and older adults with $\mathrm{AD}$ have been reported for a range of experimental paradigms, and some even report lack of significant emotional memory enhancement. For example, older adults with $\mathrm{AD}$ do not appear to benefit from emotional memory enhancement in the processing of complex information such as verbally related stories and even some short-term memory delay recognition memory paradigms (Abrisqueta-Gomez et al., 2002; Kensinger et al., 2003). These differences in patients with AD are attested when investigating both only positively and only negatively valenced stimuli (Hamann et al., 2002; Padovan et al., 2002).

Contrarily, other work indicates that even individuals with $\mathrm{AD}$ benefit modestly from emotional memory enhancement (Kensinger et al., 2002). For example, while emotional memory enhancement was attenuated relative to healthy, age-matched controls, patients with early $\mathrm{AD}$ showed improved memory for emotional items in a free recall paradigm (Nieuwenhuis-Mark et al., 2009). Indeed, some studies imply that people with AD may show enhanced flashbulb memory (i.e., vivid, visuospatially detailed memories) for events with particularly intense arousal and emotional content (Kazui et al., 2003). Other controlled work on flashbulb memories suggests that these faculties are intact in AD (Ikeda et al., 1998; Kazui et al., 2000; Moayeri et al., 2000; Budson et al., 2004). The strength of this phenomenon within individuals may be linked to amygdala mass (Mori et al., 1999; reviewed by McGaugh et al., 1996; Phelps, 2004). The work reviewed earlier in the context of valence effect differences similarly finds intact emotional memory enhancement of some form in people with $\mathrm{AD}$ (Boller et al., 2002).

These data demonstrate that different paradigms come to different conclusions about whether people with $\mathrm{AD}$ benefit from emotional memory enhancement or the positivity effect, though studies suggest these phenomena may be partially intact. To attempt to contextualize these disparate findings, we investigate a few studies in more detail.

\section{CAN EMOTIONAL MEMORY DECREMENT OCCUR?}

While most studies indicate emotional memory enhancement in older adults and some further observe a form of this in people with $\mathrm{AD}$, we have also cited work that fails to observe this phenomenon in the demented population. Borg et al. (2011) and colleagues have elucidated a model suggesting that emotional content inexorably monopolizes a certain share of executive function resources (Figure 1) based on the finding that memory deficits were greater for emotional than non-emotional stimuli when a task required more executive demand or when individuals had impaired executive resources (e.g., secondary to dementia). 


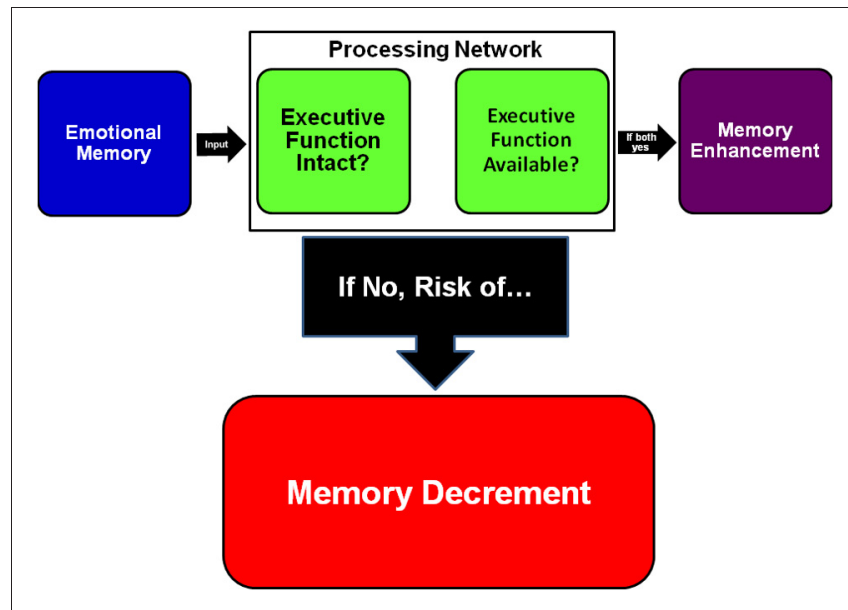

FIGURE 1 | A model based on Borg et al. (2011) and colleagues' view of how occupied or impaired cognitive resources can change how emotion affects memory encoding. If individuals' executive function resources are otherwise engaged or individuals' executive function is impaired, as in Alzheimer dementia, the model predicts greater likelihood of emotional memory decrement. Patients with intermediate executive function impairment, as in $\mathrm{MCl}$ or early $\mathrm{AD}$, would be hypothesized to have relatively normal emotional memory enhancement.

In a simple visual recognition task, younger adults, older adults, and patients with early $\mathrm{AD}$ all showed emotional memory enhancement. However, older adults and patients with early $\mathrm{AD}$ no longer showed emotional memory enhancement in a more executive function-demanding visual binding task, and patients with early $\mathrm{AD}$ also showed a between-task performance difference for non-emotional stimuli. Borg et al. (2011) and colleagues argued that emotional memory enhancement requires executive function resources and that that resource usage could either help or hinder ultimate performance depending upon total available executive function resources, a function of the individual in question, and the cognitive resources necessary for a given task.

This contextualization helps explain the disparate findings prior reviewed. Individual differences in the degree of executive dysfunction between cohorts and differences in executive function required for a given task would affect whether emotional memory enhancement, emotional memory decrement, or neither would occur in people with AD. These findings also contextualize research reports outside the dementia literature that appear at-odds with consensus elsewhere. For example, the finding that older adults more than younger adults fail to remember details of complex emotional scenes such as robberies (Aizpurua et al., 2011) may be explained in part by such stimuli having more complex, less controlled elements than the simpler stimuli normatively employed in the psychological literature.

This model greatly resembles the "dual competition" framework articulated by Pessoa (2009). That framework, based on work with younger cohorts, suggested, namely, that interactions between cognition, motivation, and emotion could affect whether emotional memory enhancement or emotional memory decrement would occur.

It is worth noting that this model does not account for the finding that patients with $\mathrm{AD}$ appear to have intact flashbulb memory. Whereas the model might predict that memory of complex events requiring concurrent executive function may suffer given emotional context, flashbulb memory, tending as it does to be associated with emotionally arousing events, shows the opposite trend. One possibility is that recollection per se is what these flashbulb memory paradigms have tested, and that recollection itself does not require extensive executive resources, even though processing the event itself may well have required them. Another possibility is that the patients with $\mathrm{AD}$ tested in these protocols have confabulated the details of the various events in ways not accounted for by the various protocols and that this confabulation masks impairment not otherwise recorded.

Concerns about the specific applicability of flashbulb memory findings aside, this model suggests that while integrating emotional content into cognitive rehabilitation for people with MCI or very early $\mathrm{AD}$ may be efficacious, it may be less useful for moreimpaired populations. Emotional memory decrement may even occur if the rehabilitation protocol in question requires intensive executive control.

We next look to neuroimaging for evidence of the neurological mechanism underlying Borg et al. (2011) and colleagues' executive resource hypothesis.

\section{TOWARD A NEURAL MECHANISM FOR BORG ET AL. (2011) AND COLLEAGUES' EXECUTIVE RESOURCE HYPOTHESIS}

Neuroimaging of emotional memory enhancement in aging and AD populations remains in its infancy. Most imaging work with patients with $\mathrm{AD}$ focuses on memory effects per se in the absence of emotional context. Nevertheless, these memory findings have revealed intriguing effects in emotional brain regions. Patients with $\mathrm{AD}$ show abnormal connectivity and activation of emotionrelated brain regions even when engaging in putatively nonemotional memory tasks (Rosenbaum et al., 2010). Specifically, increased connectivity between amygdala and pre-frontal cortex for a working memory task was seen in participants with $\mathrm{AD}$ relative to controls in spite of no differential indication of arousal correlates during the task. Rosenbaum et al. (2010) and colleagues suggest that this difference represents recruitment of compensatory pre-frontal brain structures and that the amygdala acts as an intermediary through which pre-frontal resources are accessed. During an emotionally neutral delayed match-tosample task, we found significantly more task-related amygdala

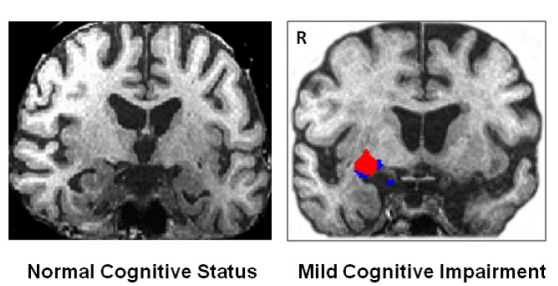

FIGURE 2 | Pilot data from Broster et al. (2012) corroborating the findings of Rosenbaum et al. (2010). We observe significant right amygdala activation during a working memory task in an older patient with mild cognitive impairment (right, white background), but not in an older control participant (left, black background). The red blob shows activation from the $Z$ map of all objects $(p<0.000001)$. 
activation in participants with MCI than in age-matched older adults (Figure 2) (Broster et al., 2012). Our findings are analogous to those reported by Rosenbaum et al. (2010).

One possible criticism of both these neuroimaging findings is that memory-impaired cohorts may experience anxiety when asked to perform a task at which they are impaired. In other words, the amygdala and frontal responses in these groups could reflect functionally irrelevant anxiety about performance. To address this concern, Rosenbaum et al. (2010) and colleagues point to a lack of arousal difference between groups and argue that that similar arousal indicates that anxiety was not responsible for the observed effects.

These findings, if valid, provide a possible neurological mechanism for emotional memory enhancement. They also provide a mechanism for Borg et al. (2011) and colleagues' claim that executive function resources, implicated as they are in pre-frontal cortical structures, determine whether emotional memory enhancement or emotional memory decrement will occur. Specifically, the pre-occupation of pre-frontal executive function resources with an executive function-intensive task could disrupt pre-frontal recruitment and explain why emotional memory enhancement fails to occur.

The finding that interaction between the amygdala and prefrontal cortices plays a significant role in implementing cognitive-emotional interactions is consistent with the amygdala's theoretical role as a coordinator of cortical functions regarding affectively significant events (Pessoa and Adolphs, 2010, but see also de Gelder et al., 2011). It is further consistent with anatomical connectivity research in the non-human primate, which purports that the relationship of the amygdala to the prefrontal cortex, and to the orbitofrontal cortex in particular, is likely implicated in emotional processing (Ghashghaei and Barbas, 2002). Nonprimate animal research also supports this conclusion (Quirk and Beer, 2006).

Future work should investigate this phenomenon while more aggressively measuring correlates of arousal and anxiety. However, even if the effect turns out not to be differentiable from presumed-functionally irrelevant task anxiety, the mechanism may nonetheless prove interesting. For example, arousal may be an epiphenomenon of the emotional memory enhancement phenomenon.

\section{SUMMARY AND RELEVANCE FOR COGNITIVE REHABILITATION}

We have reviewed evidence that patients with $\mathrm{AD}$ better remember emotional content, but only if that content is not accompanied by complex, mentally taxing phenomena. The neurological mechanism underlying this benefit may lie in the amygdala's role as an intermediary to the executive resources of the frontal cortex. We assert that the available scientific evidence suggests that patients with MCI or early AD may benefit from integrating emotional memory enhancement into the rehabilitation process. In particular, the evidence implies that coupling positively valenced stimuli to non-complex elements of the cognitive training process may be of particular use in non-depressed patients identified as having amnestic MCI or early AD. Some cohorts, such as patients with history of depression, may have attenuated positivity effects and likewise show reduced benefit from therapy. Another clinical application of Borg et al. (2011) and colleagues' model might involve using cognitive rehabilitation protocols to target executive function directly. Increased executive resources could increase patients' ability to benefit from emotional cues in their daily lives (for reviews, see Jean et al., 2010a,b or Martin et al., 2011; for a meta-analysis, see Li et al., 2010).

We have also identified two models, one behavioral (Borg et al., 2011) and one neuroaffective (Rosenbaum et al., 2010; Broster et al., 2012), capable of consolidating at the algorithmic and implementational levels, respectively, seemingly disparate reports of the interactions between emotional memory enhancement, aging, and task difficulty. Stronger tests of these findings, particularly regarding the role of the amygdala in recruiting prefrontal structures in MCI and dementia, would help confirm a promising model that describes how brain systems compensate for early cognitive impairment.

\section{REFERENCES}

Abrisqueta-Gomez, J., Bueno, O. F., Oliveira, M. G., and Bertolucci, P. H. (2002). Recognition memory for emotional pictures in Alzheimer's disease. Acta Neurol. Scand. 105, 51-54.

Aizpurua, A., Garcia-Bajos, E., and Migueles, M. (2011). False recognition and source attribution for actions of an emotional event in older and younger adults. Exp. Aging Res. 37, 310-329.

Boller, F., Dequeker, J., Degreef, H., Massioui, F. E. L., Devouche, E., Busschots, A.-M., Mallia, C., Traykov, L., Pomati, S., and Starkstein, S. E. (2002). Processing emotional information in Alzheimer's disease: effects on memory performance and neurophysiological correlates. Dement.
Geriatr. Cogn. Disord. 14, 104-112.

Borg, C., Leroy, N., Favre, E., Laurent, B., and Thomas-Antérion, C. (2011). How emotional pictures influence visuospatial binding in short-term memory in aging and Alzheimer's disease? Brain Cogn. 76, 20-25.

Broster, L. S., Gu, R., Wing, S., Guo, C., Clark, J., Heflin, M., Jicha, G., and Jiang, Y. (2012). "Increased amygdala functional connectivity during working memory among patients with mild cognitive impairment," Abstract for: OHBM 2012, the 18th Annual Meeting of the Organization for Human Brain Mapping, June 10-14, Beijing, China, Abstract 5342. Budson, A. E., Simons, J. S., Sullivan, A. L., Beier, J. S., Soloman, P. R., Scinto, L. F., Daffner, K. R., and Schacter,
D. L. (2004). Memory and emotions for the September 11, 2001, terrorist attacks in patients with Alzheimer's disease, patients with mild cognitive impairment, and healthy older adults. Neuropsychology 18, 315-327.

Burt, D. B., Zembar, M. J., and Niederehe, G. (1995). Depression and memory impairment: a metaanalysis of the association, its pattern, and specificity. Psychol. Bull. 117, 285-305.

Carstensen, L. L., Isaacowitz, D. M., and Charles, S. T. (1999). Taking time seriously: a theory of socioemotional selectivity. Am. Psychol. 54, 165-181.

Charles, S. T., Mather, M., and Carstensen, L. L. (2003). Aging and emotional memory: the forgettable nature of negative images for older adults. J. Exp. Psychol. Gen. 132, 310-324.

Charles, S. T., and Carstensen, L. L. (2007). "Emotion regulation and aging," in Handbook of Emotion Regulation, eds J. J. Gross, and R. A. Thompson (New York, NY: Guilford Press), 307-327.

de Gelder, B., van Honk, J., and Tamietto, M. (2011). Emotion in the brain: of low roads, high roads, and roads less traveled. Nat. Rev. Neurosci. 12, 425.

Evans-Roberts, C. E. Y., and Turnbull, O. H. (2011). Remembering relationships: preserved emotion-based learning in Alzheimer's disease. Exp. Aging Res. 37, 1-16.

Ghashghaei, H. T., and Barbas, H. (2002). Pathways for emotion: interactions of prefrontal and anterior temporal pathways in amygdala of 
the rhesus monkey. Neuroscience 115, 1261-1279.

Grühn, D., Scheibe, S., and Baltes, P. B. (2007). Reduced negativity effect in older adults' memory for emotional pictures: the heterogeneityhomogeneity list paradigm. Psychol. Aging 22, 644-649.

Hamann, S., Monarch, E. S., and Goldstein, F. C. (2002). Impaired fear conditioning in Alzheimer's disease. Neuropsychologia 40, 1187-1195.

Hiroaki, K. (2002). Emotion and memory: four studies of the emotional memory in Alzheimer's disease. Jpn. J. Neuropsychol. 18, 150-156.

Hopper, T. L. (2003). “They're just going to get worse anyway": perspectives on rehabilitations for nursing home residents with dementia. J. Commun. Disord. 36, 345-359.

Ikeda, M., Mori, E., Hirono, N., Imamura, T., Shimomura, T., Ikejiri, Y., and Yamashita, H. (1998). Amnestic people with Alzheimer's disease who remembered the Kobe earthquake. Br. J. Psychiatr. 172, 425-428.

Isaacowitz, D. M., Allard, E. S., Murphy, N. A., and Schlangel, M. (2009). The time course of age-related preferences toward positive and negative stimuli. J. Gerontol. B Psychol. Sci. Soc. Sci. 64, 1-5.

Jean, L., Bergeron, M.-Ẽ., Thivierge, S., and Simard, M. (2010a). Cognitive intervention programs for individuals with mild cognitive impairment: systematic review of the literature. Am. J. Geriatr. Psychol. 18, 281-296.

Jean, L., Simard, M., Wiederkehr, S., Bergeron, M. E., Turgeon, Y., Hudon, C., Tremblay, I., and van Reekum, R. (2010b). Efficacy of a cognitive training programme for mild cognitive impairment: results of a randomized controlled trial. Neuropsychol. Rehabil. 20, 377-405.

Kazui, H., Mori, E., Hashimoto, M., and Hirono, N. (2003). Enhancement of declarative memory by emotional arousal and visual memory function in Alzheimer's disease. J. Neuropsychiatry Clin. Neurosci. 15, 221-226.

Kazui, H., Mori, E., Hashimoto, M., Hirono, N., Imamura, T., Tanimukai, S., Hanihara, T., and Cahill, L. (2000). Impact of emotion on memory: a controlled study of the influence of emotionally charged material on declarative memory in Alzheimer's disease. Br. J. Psychiatr. 177, 343-347.

Kensinger, E. A. (2009). Emotional Memory Across the Adult Lifespan. New York, NY: Psychology Press.
Kensinger, E. A., Anderson, A., Growdon, J. H., and Corkin, S. (2003). Effects of Alzheimer disease on memory for verbal emotional information. Neuropsychologia 42, 791-800.

Kensinger, E. A., Brierley, B., Medford, N., Growdon, J., and Corkin, S. (2002). The effect of normal aging and Alzheimer's disease on emotional memory. Emotion 2, 118-134.

Kerestes, R., Ladouceur, C. D., Meda, S., Nathan, P. J., Blumberg, H. P., Maloney, K., Ruf, B., Saricicek, A., Pearlson, G. D., Bhagwagar, Z., and Phillips, M. L. (2012). Abnormal prefrontal activity subserving attentional control of emotion in remitted depressed patients during a working memory task with emotional distracters. Psychol. Med. 42, 29-40.

Kessels, R. P. C., and de Haan, E. H. F. (2003). Implicit learning in memory rehabilitations: a meta-analysis on errorless learning and vanishing cues methods. J. Clin. Exp. Neuropsychol. 25, 805-814.

Kessels, R. P. C., Remmerswaal, M., and Wilson, B. A. (2011). Assessment of nondeclarative Learning in severe Alzheimer dementia: the implicit memory test. Alzheimer Dis. Assoc. Disord. 25, 179-183.

Knight, M., Seymour, T. L., Gaunt, J. T., Baker, C., Nesmith, K., and Mather, M. (2007). Aging and goaldirected emotional attention: distraction reverses emotional biases. Emotion 7, 705-714.

Korczyn, A. D., and Halperin, I. (2009). Depression and dementia. J. Neurol. Sci. 283, 139-142.

Labouvie-Vief, G. (2003). Dynamic integration: affect, cognition, and the self in adulthood. Curr. Dir. Psychol. Sci. 12, 201-206.

Labouvie-Vief, G., Diehl, M., Jain, E., and Zhang, F. (2007). Six-year change in affect optimization and affect complexity across the adult life span: a further examination. Psychol. Aging 22, 738-751.

Leclerc, C. M., and Kensinger, E. A. (2011). Neural processing of emotional pictures and words: a comparison of younger and older adults. Dev. Neuropsychol. 36, 519-538.

Li, H., Li, J., Li, N., Li, B., Wang, P., and Zhou, T. (2010). Cognitive intervention for persons with mild cognitive impairment: a meta-analysis. Ageing Res. Rev. 10, 285-296.

Löckenhoff, C. E., and Carstensen, L. L. (2007). Aging, emotion, and healthrelated decision strategies: motivational manipulations can reduce age differences. Psychol. Aging 22, 134-146.

Martin, M., Clare, L., Altgassen, A. M., Cameron, M. H., and Zehnder, F (2011). Cognition-based interventions for healthy older people and people with mild cognitive impairment. Coch. Database Syst. Rev. 19 CD006220.

Mather, M., and Knight, M. (2005). Goal-directed memory: the role of cognitive control in older adults' emotional memory. Psychol. Aging 20, 554-570.

Mather, M., and Knight, M. R. (2006). Angry faces get noticed quickly: threat detection is not impaired among older adults. J. Gerontol. B Psychol. Sci. Soc. Sci. 61, 54-57.

McGaugh, J. L., Cahill, L., and Roozendaal, B. (1996). Involvement of the amygdala in memory storage: interaction with other brain systems. Proc. Natl. Acad. Sci. U.S.A. 93, 13508-13514.

Mikels, J., Larkin, G., Reuter-Lorenz, P., and Carstensen, L. (2005). Divergent trajectories in the aging mind: changes in working memory for affective versus visual information with age. Psychol. Aging 20, 542-553.

Mimura, M., and Komatsu, S. (2007) Cognitive rehabilitations and cognitive training for mild dementia. Psychogeriatrics 7, 137-143.

Moayeri, S. E., Cahill, L., Jin, Y., and Potkin, S. G. (2000). Relative sparing of emotionally influenced memory in Alzheimer's disease. Neuroreport 11, 653-655.

Mori, E., Ikeda, M., Hirono, N., Kitagaki, H., Imamura, T., and Simomura, T. (1999). Amygdalar volume and emotional memory in Alzheimer's disease. Am. J. Psychiatr. 156, 216-222.

Mowszowski, L., Batchelor, J., and Naismith, S. L. (2010). Early intervention for cognitive decline: can cognitive training be used as a selective preventive technique? Int. Psychogeriatr. 22, 537-548.

Murphy, N. A., and Isaacowitz, D. M. (2008). Preferences for emotional information in older and younger adults: a meta-analysis of memory and attention tasks. Psychol. Aging 23, 263-286.

Nashiro, K., Sakaki, M., and Mather, M. (2011). Age differences in brain activity during emotion processing: reflections of age-related decline or increased emotion regulation? Gerontology Neuropsychiatr. Clin. Neurosci. 15, 221-226.

Nieuwenhuis-Mark, R. E., Schalk, K., and de Graaf, N. (2009). Free recall and learning of emotional word lists in very elderly people with and without dementia. Am. J. Alzheimer's Dis. Other Dement. 24, 155-162.

Orgeta, V. (2011). Avoiding threat in late adulthood: testing two life span theories of adulthood. Exp. Aging Res. 37, 449-472.

Padovan, C., Versace, R., ThomasAnterion, C., and Laurent, B. (2002). Evidence for a selective deficit in automatic activation of positive information in patients with Alzheimer's disease in an affective priming paradigm. Neuropsychologia 40, 335-339.

Pessoa, L. (2008). On the relationship between emotion and cognition. Nat. Rev. Neurosci. 9, 148-158.

Pessoa, L. (2009). How do emotion and motivation direct executive control? Trends Cogn. Sci. 13, 160-166.

Pessoa, L. (2010). Emergent processes in cognitive-emotional interactions. Dialogues Clin. Neurosci. 12, 433-448.

Pessoa, L., and Adolphs, R. (2010). Emotion processing and the amygdala: from a "low road" to "many roads" of evaluating biological significance. Nat. Rev. Neurosci. 11, 773-783.

Phelps, E. A. (2004). Human emotion and memory: interactions of the amygdala and Hippocampal complex Hippocampal complex. Curr. Opin. Neurobiol. 14, 198-202.

Quirk, G. J., and Beer, J. S. (2006). Prefrontal involvement in the regulation of emotion: convergence of rat and human studies. Curr. Opin. Neurobiol. 16, 723-727.

Redondo, J., and Fernández-Rey, J. (2010). Recognition memory for emotional pictures: effects of the valence when the arousal is controlled. Psicológica 31, 65-86.

Rosenbaum, R., Furey, M., Horwitz, B., and Grady, C. (2010). Altered connectivity among emotionrelated brain regions during short-term memory in Alzheimer's disease. Neurobiol. Aging 31, 780-786.

Rozin, P., and Royzman, E. B. (2001). Negativity bias, negativity dominance, and contagion. Pers. Soc. Psychol. Rev. 5, 296-320.

Scheibe, S., and Carstensen, L. L. (2010). Emotional aging: recent findings and future trends. $J$ Gerontol. B Psychol. Sci. Soc. Sci. 65, 135-144.

van Halteren-van Tilborg, I. A., Scherder, E. J., and Hulstijn, W. (2007). Motor-skill learning in Alzheimer's disease: a review with 
an eye to the clinical practice. Neuropsychol. Rev. 17, 203-212.

Yang, L., and Hasher, L. (2011). Age differences in the automatic accessibility of emotional words from semantic memory. Cogn. Emot 25, 3-9.

Zanetti, O., Binetti, G., Magni, E., Rozzini, L., Bianchetti, A., and Trabucchi, M. (1997). Procedural memory stimulation in Alzheimer's disease: impact of a training programme. Acta Neurol. Scand. 95, 152-157.

Conflict of Interest Statement: The authors declare that the research was conducted in the absence of any commercial or financial relationships that could be construed as a potential conflict of interest.

Received: 17 January 2012; accepted: 15 March 2012; published online: 30 March 2012.

Citation: Broster LS, Blonder LX and Jiang $Y$ (2012) Does emotional memory enhancement assist the memory-impaired?. Front. Ag. Neurosci. 4:2. doi: 10.3389/fnagi.2012.00002

Copyright (c) 2012 Broster, Blonder and Jiang. This is an open-access article distributed under the terms of the Creative Commons Attribution Non Commercial License, which permits non-commercial use, distribution, and reproduction in other forums, provided the original authors and source are credited. 